\title{
Influence of chemi-ionization and chemi-recombination processes on the population of hydrogen Rydberg states in atmospheres of late type dwarfs
}

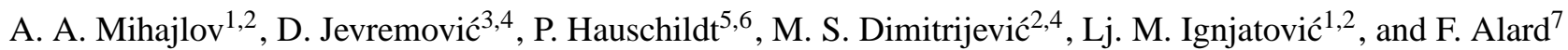 \\ 1 Institute of Physics, PO Box 57, 11001 Belgrade, Serbia and Montenegro \\ 2 Institute Isaac Newton of Chile, Yugoslavia Branch, Belgrade, Serbia and Montenegro \\ 3 APS Division, Phys. Depart., Queen's University Belfast, BT7 1NN, Northern Ireland, UK \\ ${ }^{4}$ Astronomical Observatory, Volgina 7, 11160 Belgrade, Serbia and Montenegro \\ 5 Depart. of Phys. and Astronomy and Center for Simulat. Physics, University of Georgia, Athens, GA 30602, USA \\ ${ }^{6}$ Hamburger Sternwarte, Universitaet Hamburg, Gojenbergsweg 112, 21029 Hamburg, Germany \\ 7 CRAL/ENS, 46 Allée d'Italie, 69634 Lyon Cedex 07, France
}

Received 22 July 2002 / Accepted 4 February 2003

\begin{abstract}
We study the influence of a group of chemi-ionization and chemi-recombination processes on the populations of higher states of hydrogen in the layers of a stellar atmosphere. The group of processes includes ionization: $\mathrm{H}^{*}(n)+\mathrm{H}(1 \mathrm{~s}) \Longrightarrow$ $\mathrm{H}_{2}^{+}+\mathrm{e}, \mathrm{H}^{*}(n)+\mathrm{H}(1 \mathrm{~s}) \Longrightarrow \mathrm{H}(1 \mathrm{~s})+\mathrm{H}^{+}+\mathrm{e}$, and inverse recombination: $\mathrm{H}_{2}^{+}+\mathrm{e} \Longrightarrow \mathrm{H}^{*}(n)+\mathrm{H}(1 \mathrm{~s}), \mathrm{H}(1 \mathrm{~s})+\mathrm{H}^{+}+\mathrm{e} \Longrightarrow \mathrm{H}^{*}(n)+\mathrm{H}(1 \mathrm{~s})$, where $\mathrm{H}^{*}(n)$ is the hydrogen atom in a state with the principal quantum number $n \gg 1$, and $\mathrm{H}_{2}^{+}$is the hydrogen molecular ion in a weakly bound rho-vibrational state of the ground state. These processes have been treated within the framework of the semi-classical approximation, developed in several previous papers, and have been included in the general stellar atmosphere code PHOENIX. We present results for an $\mathrm{M}$ dwarf atmosphere with $T_{\mathrm{eff}}=3800 \mathrm{~K}$ and find that the inclusion of chemi-ionization and chemi-recombination processes is significant in the low temperature parts of the atmosphere.
\end{abstract}

Key words. atomic processes - molecular processes - stars: late type

\section{Introduction}

For several years we have studied the chemi-ionization atomatom collisional processes and inverse chemi-recombination processes that may influence the populations of the hydrogen atom Rydberg states in the weakly ionized layers of stellar atmospheres (Mihajlov et al. 1996a, 1997). In the case of hydrogen, these processes are:

$\mathrm{H}^{*}(n)+\mathrm{H}(1 \mathrm{~s}) \Longrightarrow \mathrm{H}_{2}^{+}+\mathrm{e}$,

$\mathrm{H}^{*}(n)+\mathrm{H}(1 \mathrm{~s}) \Longrightarrow \mathrm{H}(1 \mathrm{~s})+\mathrm{H}^{+}+\mathrm{e}$,

$\mathrm{H}_{2}^{+}+\mathrm{e} \Longrightarrow \mathrm{H}^{*}(n)+\mathrm{H}(1 \mathrm{~s})$

$\mathrm{H}(1 \mathrm{~s})+\mathrm{H}^{+}+\mathrm{e} \Longrightarrow \mathrm{H}^{*}(n)+\mathrm{H}(1 \mathrm{~s})$,

where $\mathrm{H}^{*}(n)$ is a hydrogen atom in a state with the principal quantum number $n \gg 1$, and $\mathrm{H}_{2}^{+}$is a hydrogen molecular ion in a weakly bound rho-vibrational state of the ground electronic state. In the case of ionization $(1 a, b)$, the lower limit of the Rydberg state range that was considered was set to $n=4$ (Mihajlov et al. 1996a, 1997). The block of rho-vibrational

Send offprint requests to: M. S. Dimitrijević, e-mail: mdimitrijevic@aob.bg.ac.yu states with a dissociation energy less than $1 \mathrm{eV}$ has an essential role in the case of formation of the molecular ion (processes (1a) and (2a)) and within the range of quantum numbers that was considered. In the case of cool stellar atmosphere layers $(3000 \mathrm{~K} \leq T \leq 5000 \mathrm{~K})$, the block of such weakly bound rho-vibrational states can be considered to be in equilibrium with the collisional $\mathrm{H}(1 \mathrm{~s})+\mathrm{H}^{+}$complexes, without the requirement that the lower rho-vibrational states of molecular ion are in equilibrium (Mihajlov \& Ljepojević 1982; Mihajlov et al. 1992). This fact makes the description of recombination processes (2a) and ( $2 b$ ) easier, since it enables us to avoid the direct determination of the density of molecular ions in weakly ionized rho-vibrational states.

In our previous work (Mihajlov et al. 1997, 1998) we estimated the influence of the processes $(1 a, b ; 2 a, b)$ for the low temperature layers of the Solar atmosphere. We compared the ionization and recombination fluxes with and without inclusion of the above processes and found a significant difference in the population of the $\mathrm{H}^{*}(n)$ atomic states with $4<n<8$.

Here we discuss the importance of these processes in stellar atmospheres of lower temperature. This paper is organized as follows: in Sect. 2 we summarize the theoretical framework and in Sect. 3 we describe the changes in the stellar atmosphere 
code PHOENIX and parameters of the test model atmosphere. We present our results and discussion in Sect. 4 and draw our conclusions in Sect. 5.

\section{Theoretical remarks}

Let $I_{\mathrm{i}}^{(\mathrm{a}, \mathrm{b})}(n)$ and $I_{\mathrm{r}}^{(\mathrm{a}, \mathrm{b})}(n)$ denote ionization and recombination fluxes affecting population and depopulation of excited hydrogen atom atomic levels due to reactions. By definition, ionization fluxes $I_{\mathrm{i}}^{(\mathrm{a}, \mathrm{b})}(n)$ determining the rate of depopulation of $\mathrm{H}^{*}(n)$ states are given by

$I_{\mathrm{i}}^{(\mathrm{a})}(n)=K_{i}^{(\mathrm{a})}(n) \cdot N(\mathrm{H}) \cdot N\left(\mathrm{H}^{*}(n)\right)$

$I_{\mathrm{i}}^{(\mathrm{b})}(n)=K_{i}^{(\mathrm{b})}(n) \cdot N(\mathrm{H}) \cdot N\left(\mathrm{H}^{*}(n)\right)$

where $N(\mathrm{H})$ and $N\left(\mathrm{H}^{*}(n)\right)$ are $\mathrm{H}(1 \mathrm{~s})$ and $\mathrm{H}^{*}(n)$ atom densities, and $K_{\mathrm{i}}^{\mathrm{a}}(n)$ and $K_{\mathrm{i}}^{\mathrm{b}}(n)$ are rate coefficients of the reactions (1a) and (1b) respectively. The recombination flux $I_{\mathrm{r}}^{(\mathrm{a})}(n)$, determining the velocity of population of $\mathrm{H}^{*}(n)$ states due to the reaction $(2 \mathrm{a})$, is given by definition as: $I_{\mathrm{r}}^{(\mathrm{a})}=K_{\mathrm{dr}} \cdot N\left(\mathrm{H}_{2}^{+}\right) \cdot N(\mathrm{e})$, where $N(\mathrm{e})$ and $N\left(\mathrm{H}_{2}^{+}\right)$are the electron density and the density of molecular ion $\mathrm{H}_{2}^{+}$in the abovementioned weakly bound rhovibrational states, and $K_{\mathrm{dr}}$ is the corresponding rate coefficient of the dissociative recombination reaction (2a). The expression for the recombination flux $I_{\mathrm{r}}^{(\mathrm{a})}$ may be written by:

$I_{\mathrm{r}}^{(\mathrm{a})}(n)=K_{\mathrm{r}}^{(\mathrm{a})}(n) \cdot N(\mathrm{H}) \cdot N\left(\mathrm{H}^{+}\right) \cdot N(\mathrm{e})$

where $K_{\mathrm{r}}^{(\mathrm{a})}=K_{\mathrm{dr}}\left[N(\mathrm{H}) \cdot N\left(\mathrm{H}^{+}\right) / N\left(\mathrm{H}_{2}^{+}\right)\right]^{-1}$. The advantage of expression (4a) for the recombination flux $I_{\mathrm{r}}^{(\mathrm{a})}(n)$ is the fact that it introduces a new effective rate coefficient of the reaction (2a). If the ionization rate coefficient $K_{\mathrm{i}}^{\mathrm{a}}(n)$ is known, the $K_{\mathrm{r}}^{(\mathrm{a})}(n)$ may be determined directly, under assumption of the equilibrium between $\mathrm{H}_{2}^{+}$weakly bound rho-vibrational states and the $\mathrm{H}+\mathrm{H}^{+}$ collisional complex (Mihajlov \& Ljepojević 1982; Mihajlov et al. 1992). This makes the treatment of the problem easier and enables us to avoid the determination of the $K_{\mathrm{dr}}(n)$ rate coefficient. The recombination flux $I_{\mathrm{r}}^{(\mathrm{b})}(n)$, determining the rate of population of $\mathrm{H}^{*}(n)$ states due to the reaction $(2 \mathrm{~b})$, is given, by definition, by:

$I_{\mathrm{r}}^{(\mathrm{b})}(n)=K_{\mathrm{r}}^{(\mathrm{b})}(n) \cdot N(\mathrm{H}) \cdot N\left(\mathrm{H}^{+}\right) \cdot N(\mathrm{e})$,

where $K_{\mathrm{r}}^{(\mathrm{b})}(n)$ is the rate coefficient of the reaction $(2 \mathrm{~b})$. The influence of the processes $(1 \mathrm{a}, \mathrm{b})$ and $(2 \mathrm{a}, \mathrm{b})$ on $\mathrm{H}^{*}(n)$ atomic states populations may be estimated using only the $N(\mathrm{H})$, $N\left(\mathrm{H}^{*}(n)\right), N\left(\mathrm{H}^{+}\right)$and $N(\mathrm{e})$ populations.

Rate coefficients for the processes $(1,2)$ have been determined within the semi-classical theory described in the previous papers (Janev \& Mihajlov 1980; Mihajlov \& Ljepojević 1982; Mihajlov et al. 1992). This theory takes into account atomic collisions, where the $\mathrm{H}^{*}(n)+\mathrm{H}$ system in the processes $(1 \mathrm{a}, \mathrm{b})$ as well as the $\mathrm{e}+\mathrm{H}^{+}+\mathrm{H}$ system in the processes $(2 b)$ go through the phase where they may be treated as a $\left(\mathrm{H}^{+}+\mathrm{H}\right)+\mathrm{e}$ system. Here, $\left(\mathrm{H}^{+}+\mathrm{H}\right)$ denotes that the ion-atom subsystem may be treated as a quasi-molecular ionatom complex under assumption that its electronic states may be described as superpositions of the ground and the first excited states of the $\mathrm{H}_{2}^{+}$molecular ion. Furthemore, this means that during the quasi-molecular phase the average electron distance from the center of mass of the $\mathrm{H}^{+}+\mathrm{H}$ subsystem is much larger than the internuclear distance between $\mathrm{H}^{+}$and $\mathrm{H}$. Consequently, the dipole interaction between the $\left(\mathrm{H}^{+}+\mathrm{H}\right)$ and an electron (Smirnov \& Mihajlov 1971) is the origin of the chemi-ionization/recombination processes $(1 \mathrm{a}, \mathrm{b})$ and $(2 \mathrm{a}, \mathrm{b})$. One can notice that within the frame of this approach, the processes (2a) and (2b) may be described practically in the same way. Expressions for the rate coefficients in the processes $(1 \mathrm{a}, \mathrm{b})$ and $(2 a, b)$ which are used here as well as the method of determination of the rate coefficients are described in details in Mihajlov et al. (1996b).

\section{Modeling}

We included procesess $(1 \mathrm{a}, \mathrm{b})$ and $(2 \mathrm{a}, \mathrm{b})$ into the general stellar atmosphere code PHOENIX described in Hauschildt et al. (1999), Short et al. (1999), Baron \& Hauschildt (1998). PHOENIX solves simultaneously the equations of hydrostatic equilibrium, multi-level and multi-species statistical equilibrium and NLTE radiative transfer. The major advantage of PHOENIX is that it uses extensive atomic and molecular databases and can be used for modeling of very cool objects.

The main change in the code is in the addition of a new term in the statistical equilibrium equation which describes additional collisional processes. This change allows us to treat chemi-ionization/recombination processes consistently with all the other processes. Due to limits of the semi-classical approach, we include additional collisional term only for levels larger than $n=4$.

We use for our calculation the plane-parallel, static option of PHOENIX which has been adopted for use in "chromospheric" type of problems. As a test atmosphere we use a "schematic" chromospheric structure with an effective temperature of $3800 \mathrm{~K}$, logarithm gravity of $5.0, \log m_{0}=-2.5$ being the column mass at temperature minimum, and $\log m_{\mathrm{TR}}-4.8$, $m_{\mathrm{TR}}$ standing for the column mass in the transition region. The choosen parameters represent a typical M0 dwarf of weak activity. The procedure of building this model is identical to that described in Jevremović et al. (2000) and its structure is shown in Fig. 1. As we are interested in behavior of hydrogen populations, we solve the NLTE problem for the 30 lowest levels. The standard collisional data for hydrogen are those from Vriens \& Smeets (1980).

\section{Results and discussion}

In Figs. 2-4 we present the behavior of population ratios $\zeta$ without and with inclusion of chemi-ionization $(1 \mathrm{a}, \mathrm{b})$ and chemi-recombination $(2 \mathrm{a}, \mathrm{b})$ processes for the levels 3 to 20. The ratios are close to unity for $n=3$, and start to differ for $n=4$ and 5 (Fig. 2). For $n=5$ the largest difference is around $10 \%$. This is to be expected as we include the procesess for $n \geq 4$, which is based on the validity of the semi-classical approximation. The population of lower levels $(n=2,3)$ is influenced by other processes included in the statistical equilibrium equation. 


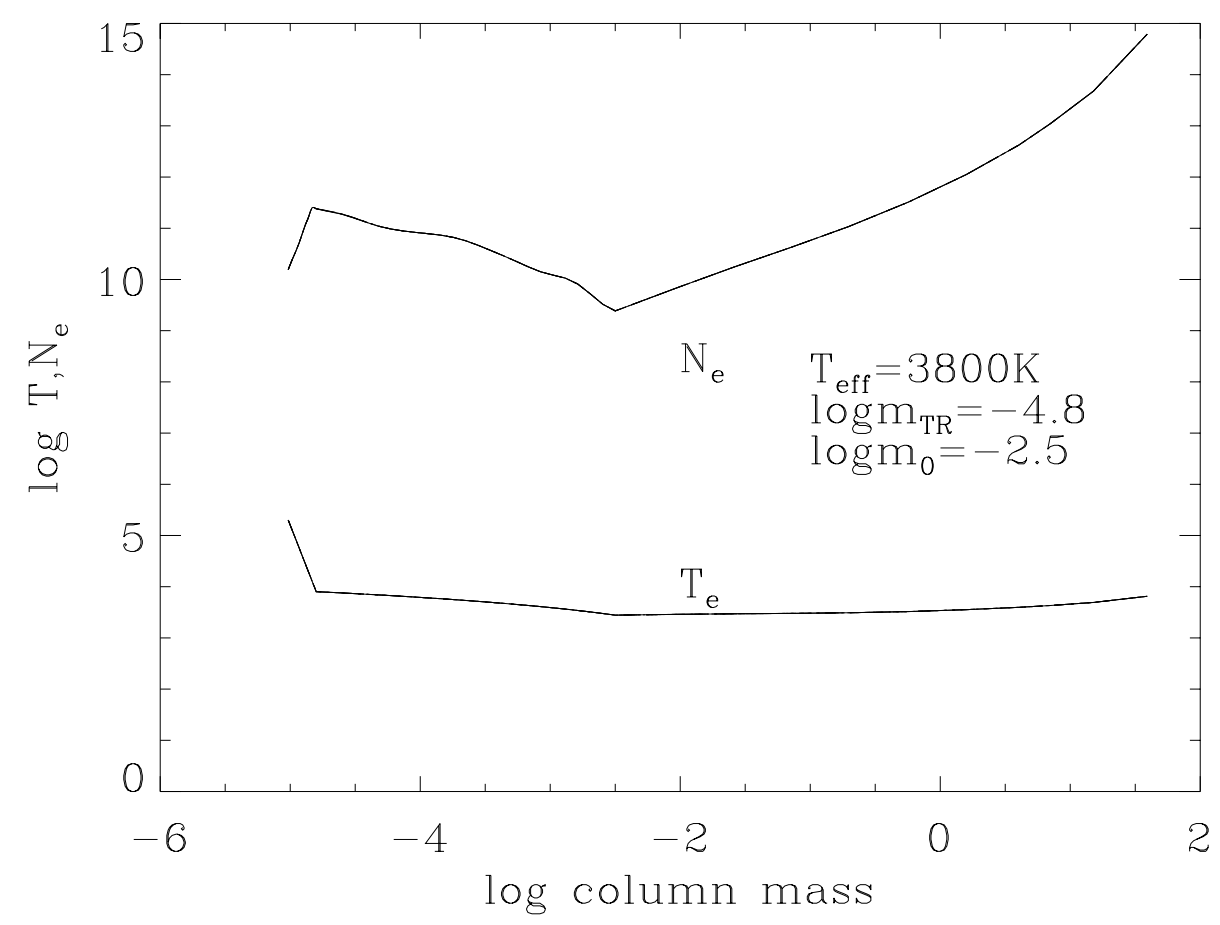

Fig. 1. Temperature and electron density structure for the model of test atmosphere.

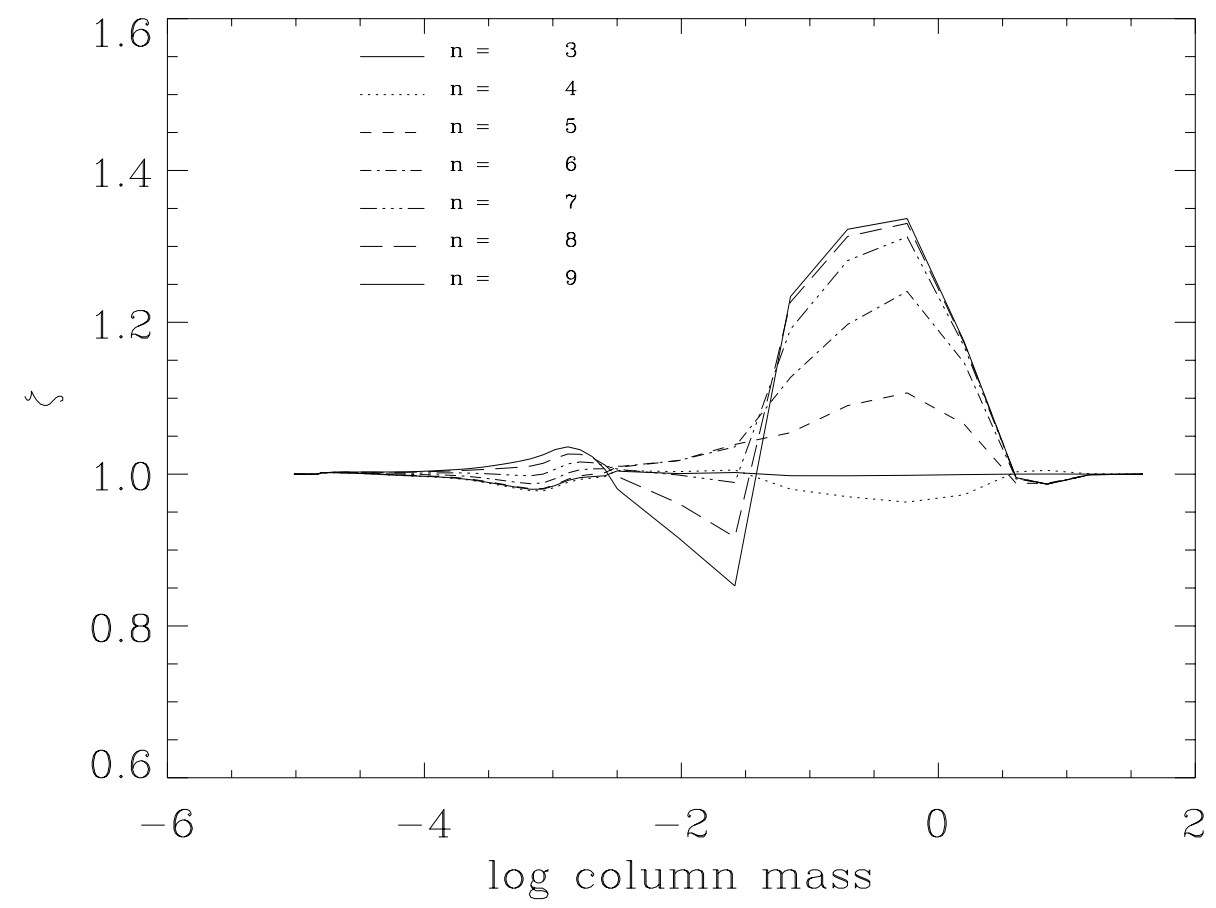

Fig. 2. The behaviour of the population ratio $\zeta(n)$ for $3 \leq n \leq 9$ as a function of the column mass.

As expected, the additional collisional term in statistical equilibrium brings the solution closer to local thermodynamical equilibrium (LTE). In terms of our population ratios, in the parts of atmosphere where populations, determined in our calculations using PHOENIX, are larger than LTE populations (departure coefficients $b_{i}>1$ ), our parameter $\zeta$ is larger than one. This means that in such a case, chemi-ionization processes depopulate excited states and dominate in comparison with chemi-recombination processes. The result is that populations determined with processes $(1,2)$ included are smaller in comparison with populations calculated without them and consequently $\zeta$ is larger than one. In the reverse case $\left(b_{i}<1\right)$, parameter $\zeta$ is smaller than one, which means that in this case chemi-recombination processes dominate over chemi-ionization ones and increase the population of excited levels. As a result, in both cases, processes $(1,2)$ act as important factors closing the difference between the calculated and LTE populations. 


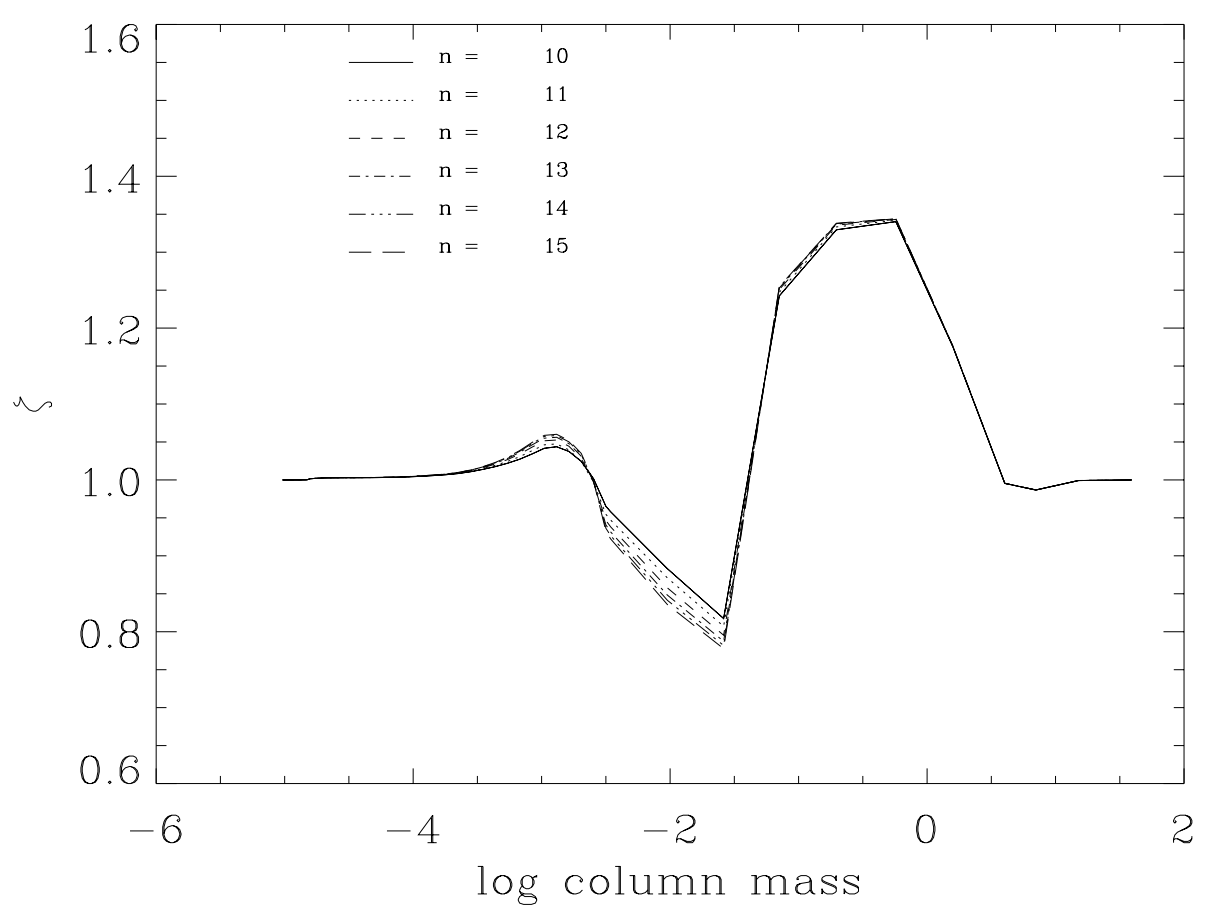

Fig. 3. The behaviour of the population ratio $\zeta(n)$ for $10 \leq n \leq 15$ as a function of the column mass.

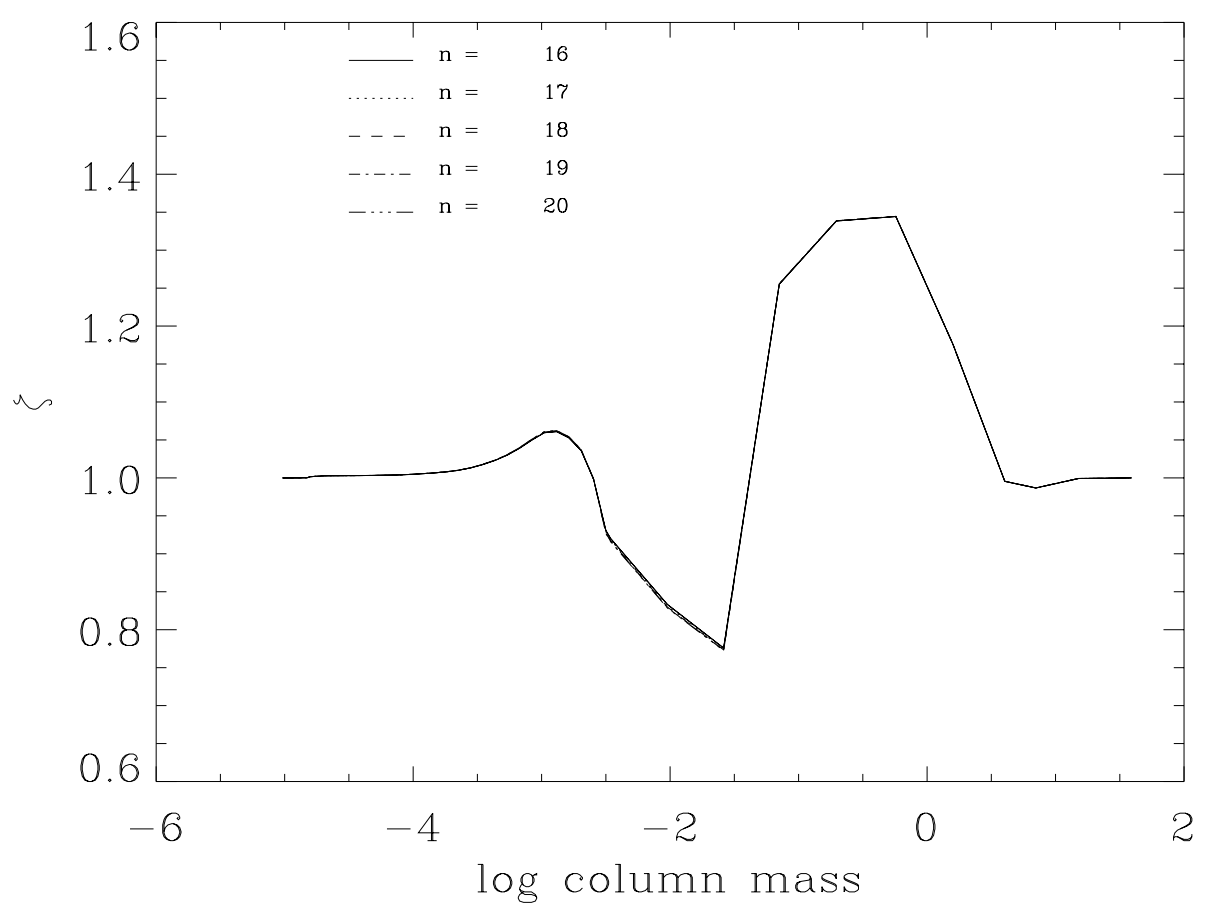

Fig. 4. The behaviour of the population ratio $\zeta(n)$ for $16 \leq n \leq 20$ as a function of the column mass.

As can be seen from Fig. 2, the behaviour of $\zeta(n)$ for $6<$ $n<9$ shows a tendence to converge to a pattern, which may differ up to $40 \%$ from unity. For higher levels (Figs. 3 and 4) the convergence to a pattern is complete and it can be understood by assuming that highly excited hydrogen level populations have quasi-Boltzman distributions different from distributions for partial LTE. It is not suprising that the population ratios for the levels up to 30 and the proton density show the same behaviour as levels with $n>15$. One also has to remember that in the layers with temperatures lower than $4500 \mathrm{~K}$ the main electron contributors are metals so, as expected, there is very little change in electron density.

\section{Conclusion}

We have demonstrated the importance of the inclusion of chemi-ionization $(1 \mathrm{a}, \mathrm{b})$ and chemi-recombination $(2 \mathrm{a}, \mathrm{b})$ processes in modeling of atmospheres of late type stars. 
In previous work (Mihajlov et al. 1996a, 1997, 1998), it had been assumed that these processes were important up to $n=10$. Here we have demonstrated that inclusion of processes $(1 \mathrm{a}, \mathrm{b})$ and $(2 a, b)$ significantly changes the population of all Rydberg levels due to PLTE. However, in some atmospheric layers selfconsistent modeling shows differences of up to $40 \%$ between calculations with and without inclusion of these processes.

We plan to continue these investigations for atmospheres with different effective temperatures, including stars with higher $T_{\text {eff }}$, such as the Sun and Sun-like stars. Namely, on the basis of our previous results and those presented here, one could expect that for such stars, processes $(1 \mathrm{a}, \mathrm{b})$ and $(2 \mathrm{a}, \mathrm{b})$ should influence not only the population of higher levels of hydrogen, but also the electron density. Furthermore, we plan to include in PHOENIX the chemi-ionization/recombination processes similar to $(1 \mathrm{a}, \mathrm{b})$ and $(2 \mathrm{a}, \mathrm{b})$ in the $\mathrm{He}\left(1 \mathrm{~s}^{2}\right)$ atom which would be used in modeling of helium-rich stars.

Also, there is a need for a developement of a theory of processes similar to processes $(1 \mathrm{a}, \mathrm{b})$ and $(2 \mathrm{a}, \mathrm{b})$ for the lower levels of hydrogen, with the principal quantum numbers $n=2$ and 3. This extension would enable more sophisticated modeling of stellar atmospheres as the population of lower levels may significantly alter the electron density and consequently the atmospheric structure.

Acknowledgements. This work is a part of the projects "Radiation and transport properties of the non-ideal laboratory and ionospheric plasma" (Project number 1466) and "Influence of collisional processes on astrophysical plasma lineshapes" (Project number GA 1195) and was supported by the Ministry of Science, Technologies and Development of Serbia. D.J. was supported at QUB through the PPARC grant PPA/G/S/1998/00034. Authors acknowledge the care of reviewer to improve this paper.

\section{References}

Baron, E., \& Hauschildt, P. H. 1998, ApJ, 495, 370

Hauschildt, P. H., Allard, F., \& Baron, E. 1999, ApJ, 512, 377

Janev, R. K., \& Mihajlov, A. A. 1980, Phys. Rev., 21, 819

Jevremović, D., Doyle, J. G., \& Short, C. I. 2000, A\&A, 358, 575

Mihajlov, A. A., \& Ljepojević, N. N. 1982, Proc. Symp. Phys. Ioniz. Gases, Dubrovnik, Contributed Papers, p. 385

Mihajlov, A. A., Ljepojević, N. N., \& Dimitrijević, M. S. 1992, J. Phys. B: At. Mol. Phys., 25, 5127

Mihajlov, A. A., Dimitrijević, M. S., Ignjatović, Lj. M., \& Vasilijević, M. M. 1996a, Int. Conf. in the memory of I. S. Shklovsky, S. A. Kaplan, S. B. Pikel'ner: Current problems in Astrophysics, Moscow, Russia, 23-28.09.1996

Mihajlov, A. A., Dimitrijević, M. S., \& Djurić, Z. 1996b, Phys. Scr., 33, 159

Mihajlov, A. A., Ignjatović, Lj. M., Vasilijević, Lj. M., \& Dimitrijević, M. S. 1997, A\&A, 324, 1206

Mihajlov, A. A., Ignjatović, Lj. M., \& Dimitrijević, M. S. 1998, Novyj cikl aktivnosty Solnca, Trudy, Sankt Peterburg, Pulkovo, p. 52

Short, C. I., Hauschildt, P. H., \& Baron, E. 1999, ApJ, 525, 375

Smirnov, V. A., \& Mihajlov, A. A. 1971, Opt. Spektrosk., 30, 984; Opt. Spectrosc., 30, 525

Vriens, L., \& Smeets, A. H. M. 1980, Phys. Rev. A, 22, 940 